\title{
Comparison of Different Media for Germ Tube Production by Candida albicans: A Retrospective Study
}

\author{
Aditi Mehta*, Mritunjay Kumar, Upasana Bhumbla, Anamika Vyas and A.S. Dalal \\ Department of Microbiology, Geetanjali Medical College and Hospital, Udaipur, India
}

*Corresponding author

\section{A B S T R A C T}

\begin{tabular}{|l|}
\hline K e y w o r d s \\
Germ tube production, \\
$\begin{array}{l}\text { Candida albicans, } \\
\text { Retrospective study }\end{array}$ \\
\hline Article Info \\
\hline $\begin{array}{l}\text { Accepted: } \\
\text { 04 May } 2018 \\
\text { Available Online: } \\
\text { 10 June } 2018\end{array}$ \\
\hline
\end{tabular}

Systemic candidiasis is associated with a high crude mortality rate, even with first line antifungal therapy. C. albicans is the predominant cause of invasive fungal diseases which is a serious public health issue. The main objective is to assess the reliability of different media for germ tube production in Candida albicans isolated from various clinical samples. All Candida isolates were identified and speciated by conventional methods such as Gram's staining, germ tube test, chlamydospore formation on corn meal agar, sugar fermentation test, sugar assimilation test, and growth on Hi-chrome candida agar. Out of 128 clinical isolates of Candida albicans, 5 different methods were used for germ tube production. Pooled human sera showed 121/128 (94.53\%) was the most sensitive method wherein YEPD (Yeast Extract Peptone Dextrose) Broth 104/128 (81.25\%) was the reliable and easy method for detection of germ tube, followed by Trypticase soy broth 93/128 (72.65\%); Peptone water 79/128 (61.71\%) and 2\% Sucrose 76/128 (59.37\%).

\section{Introduction}

The incidence and prevalence of invasive fungal infections have increased in the last decade, especially due to large population of immunocompromised patients and/or those hospitalized with serious underlying diseases. Indeed, Candida spp. are the fourth most common cause of hospital-acquired systemic infections in India with crude mortality rates of up to $30 \%$. In healthy individuals this colonization generally remains benign. $C$. albicans can cause two major types of infections in human's superficial infections, such as oral or vaginal candidiasis, and lifethreatening systemic infections. Indeed, HIV is a major risk factor for developing oral candidiasis (Ruhnke, 2006). Systemic candidiasis is associated with a high crude mortality rate, even with first line antifungal therapy. Both neutropenia and damage of the gastrointestinal mucosa are risk factors for the development of systemic (disseminated) candidiasis. Further risk factors include central venous catheters, which allow direct access of the fungus to the bloodstream, the application of broad-spectrum antibacterials, which enable fungal overgrowth, and trauma or gastrointestinal surgery, which disrupts mucosal barriers (Sachin et al., 2012).

C. albicans is the predominant cause of invasive fungal infections and represents a serious public health challenge with increasing 
medical and economic importance due to the high mortality rates and increased costs of care and duration of hospitalization. During both superficial and systemic infection (Sullivan et al., 1998), C. albicans relies on a battery of virulence factors and fitness attributes. One such suggested contributory virulence factor in the pathogenesis of $C$. albicans is germ tube. Also it is a presumptive clinical identification of $C$. albicans usually made on the basis of its ability to produce short, slender, tube-like structures called germ tubes when incubated at $35^{\circ} \mathrm{C}$ to $37^{\circ} \mathrm{C}$ for 2 to $4 \mathrm{~h}$ in pooled human serum (Taschdjian et al., 1960). The germ tube has parallel walls and no constriction at the point of origin at the blastospore mother cell. The use of human serum routinely for culture and microscopic examination of $C$. albicans in the germ tube testis cheap but presents a hazard for transmission of diseases. This study investigated the possibility of using other four different substrates for induction of germ tube by $C$. albicans and to compare their efficacy with pooled human serum in routine laboratory.

The aim of the study was to assess the reliability of different media for germ tube production in Candida albicans, in various clinical samples.

\section{Materials and Methods}

During the study period of two years, a total of 128 C. albicans strains were isolated from various clinical samples received in Microbiology department, of a tertiary care teaching hospital. All Candida isolates were identified and speciated by conventional methods such as Gram's staining, germ tube test, chlamydospore formation on corn meal agar, sugar fermentation test, sugar assimilation test, and growth on Hi-chrome candida agar (Himedia, Mumbai) (Sachin C. Deorukhkar et al., 2012)
For germ tube test substrates that were employed for induction of germ tube in clinical isolates of $C$. albicans in the present study were Trypticase soy broth, YEPD (yeast extract peptone dextrose) broth, Brain heart infusion, 2\% Sucrose, peptone water were evaluated and compared with regular substrate employing pooled human sera (Arora et al., 2003). All C. albicans isolates were subcultured onto Sabouraud's dextrose agar and were incubated at $37^{\circ} \mathrm{C}$ for $24-48$ hours before performing the germ tube test. For the germ tube test, a light inoculums was done, of 2-3 colonies of each isolate from fresh culture in $0.5 \mathrm{ml}$ of all the above media which were dispensed in $12 \times 75 \mathrm{~mm}$ test tube. A positive control (C. albicans ATCC 10231) and a negative control ( $C$. krusei) were used with each batch of yeast tested (Sachin C Deorukhkar et al., 2012). Then the inoculated test tubes were incubated at $37^{\circ} \mathrm{C}$ in a water bath for 3 hours. Evaluation of Germ tube formation was done by placing a drop of incubated suspension placed on a glass slide and covered with coverslip. Microscopic examination was done under magnification of 40X for the presence of germ tube (Kim et al., 2002). Of typical $C$. albicans reveals thin germ tubes 3 to $4 \mathrm{~mm}$ in diameter and up to 20 mm long; unlike pseudohyphae that are not constricted at their point of origin. A criterion for germtube positivity was observation of minimum five germ tubes in entire wet mount preparation. Negative results were confirmed by examining at least 10 high power fields for the presence of germ tubes (Sheppard et al., 2002).

\section{Results and Discussion}

In the present study, the germ tube production for 128 C. albicans isolates were seen by using five different substrates. Pooled human sera showed 121/128 (94.53\%), YEPD (yeast extract peptone dextrose) Broth 104/128 
(81.25\%); Trypticase soy broth 93/128 (72.65\%); Peptone water 79/128 $(61.71 \%)$ and $2 \%$ Sucrose 76/128 (59.37\%). YEPD broth and pooled human serum were performed almost similar for the induction of germ tube in C. albicans.

The incidence of candidiasis continues to rise in proportion to the growing number of patients at risk (Gopee E. Makwana et al., 2012). Thus rapid identification of Candida isolates to the species level in the clinical laboratory has become important. Several methods for the identification of yeasts have been developed. However, most of these techniques are labour-intensive technologies and expensive those are not commonly available in routine microbiology laboratory services (Sachin et al., 2012).

The germ tube test has been a long wellestablished, rapid and highly reliable presumptive test for identification of medically important C. albicans. This technique is a simple, cheap method and may therefore a favoured method for laboratories trying to work economically (Maria-Jesus Sevilla and Odds, 1986). C. albicans cells reproduce normally by budding, and they frequently produce germ tubes under unfavourable conditions (Sachin C. Deorukhkar et al., 2012). The formation of unconstricted filaments in response to serum is the basis of the 'germ tube test', to distinguish C. albicans from other Candida species; although C.dubliniensis, the nearest relative to
C. albicans, also forms unconstricted hyphae in this test. C. tropicalis after an extended incubation period of three hours may also produce germ tube-like structures (Sheppard et al., 2002).

The classical method of pooled human sera has been widely used by laboratories for several years and had a sensitivity range of $91 \%$ - 100\%. The results obtained by this study are in agreement with this parameter (sensitivity $94.53 \%$ ).

In spite of its low cost and easiness, the use of human serum for this test may have several disadvantages for example; serum has to be fresh otherwise frozen serum at $4^{\circ} \mathrm{C}$ for 15 days may have $50 \%$ decrease in germ tube production, false negative result due to the effect of biological inhibitors present in it, the yeast inoculum has to contain $<10^{7}$ cells $\mathrm{mL}^{-}$ 1 , different batches of serum may produce different results and most importantly the possible risk of biohazard (Pendru Raghunath and Seshu Kumari, 2014).

In an attempt to overcome these drawbacks, several investigators have proposed other media such as animal serum, plasma, peptone water, trypticase soya broth (TSB), Sabouraud broth, brain-heart infusion broth (BHI) (Antonella Souza et al., 2014), RPMI-1640 broth, egg white and saliva. These media, however, have low sensitivity. As compared to other media; serum is more sensitive for germ tube production.

Table.1 Sensitivity of different substrate in comparison to CHROMagar

\begin{tabular}{|l|c|}
\hline \multicolumn{1}{|c|}{ Substrate } & Sensitivity (\%) \\
\hline Pooled human sera & $94.53 \%$ \\
\hline YEPD Broth & $81.25 \%$ \\
\hline Trypticase soy broth & $72.65 \%$ \\
\hline Peptone water & $61.71 \%$ \\
\hline 2\% Sucrose & $59.37 \%$ \\
\hline
\end{tabular}


In this study, pooled human serum had highest sensitivity (94.53\%) and the possible reason may be due to the inhibitors present in the human serum, yeast cell concentration and storage condition of serum. Further, YEPD medium had sensitivity $(81.25 \%)$ and also Kim et al., (2002) reported $100 \%$ positivity at $39^{\circ}$, comparing germ tube induction in rabbit serum at $37^{\circ} \mathrm{C}$.The possible cause of variability in germ tube positivity rate may be attributed to the incubation temperature and time. (Sachin C. Deorukhkar et al., 2012; Kim et al., 2002; Atalay et al., 2017)

Trypticase soya broth had a sensitivity rate of $72.65 \%$ in our study; which is similar to the findings of (Arora et al., 2003) and (Gopee E. Makwana et al., 2012). In contrast, Joshi KR et al., and (Sachin C. Deorukhkar et al., 2012) had reported a higher sensitivity rates of $100 \%$ and 94\%respectively in Trypticase soya broth. In this study, $61.71 \%$ of $C$. albicans isolates showed germ tube test positive in peptone water. Similarly, (Sachin C. Deorukhkar et al., 2012) also reported sensitivity of $69 \%$ in peptone water.Among the less suited medium Germ tube production in $2 \%$ Sucrose solution was only $59.37 \%$; but (Pendru Raghunath and Seshu Kumari, 2014) reported a higher sensitivity rate of $80 \%$.This may be due to initial $\mathrm{pH}$ which have allowed germ-tube formation to occur and later a drop in $\mathrm{pH}$ could suppress germ-tube formation (Pollack and Hashimoto, 1987) (Table 1).

Germ tube formation in $C$. albicans is an endotrophic process, with new factors which affect germ tube formation, and the complex interrelationships between the many environmental factors.

YPED broth is found to be a better serum free substrate for germ tube production and subsequently for the presumptive differentiation of $C$. albicans from nonalbicans candida, without the extensive time required for the preparation and testing of pooled human serum. Furthermore, this medium is commercially available and is more stable, effective, and is not bio hazardous.

\section{References}

Antonella Souza, Sydney Hartz, Cecilia Bittencourt, Luciana da Silva, Flavio de Mattos and Luiz Carlos. Use of Mueller-Hinton broth and agar in the germ tube test. Rev. Inst. Med. Trop. Sao Paulo; (2014) 56 (6): 483-485, November - December

Arora DR, Saini S, Aparna, Gupta N. Evaluation of germ tube test in various media. Indian J Pathol Microbiol 2003; 46 (1): 124-126.

Atalay MA, Koc AN, Parkan OM, Aydemir G, Elmali F, Sav H. Can serums be replaced by Mueller- Hinton agar in germ tube test? Nigerian journal of clinical practice. 2017; 20(1): 61-3.

Gopee E. Makwana, Hiral Gadhavi, Mala Sinha. Comparison of germ tube production by Candida albicans in various media. NJIRM 2012; vol. 3 (2). April-June.

Kim D, Shin WS, Lee KH, Kim K, Park JY, Koch CM. Rapid differentiation of Candida albicans from Candida Species using its unique germ tube formation at $39^{\circ} \mathrm{C}$ Yeast 2002; 19: 957-962.

Maria-Jesus Sevilla and F. C. Odds. Development of Candida albicans Hyphae in different growth media variations in growth rates, cell dimensions and timing of morphogenetic events. Journal of General Microbiology (1986), 132, 3083-3088.

Pendru Raghunath, K. and Seshu Kumari. SST broth, a new serum free germ tube induction medium for identification of 
Candida albicans. World J Microbiol Biotechnol (2014) 30: 1955-1958.

Pollack JH, and Hashimoto T. The role of glucose in the $\mathrm{pH}$ regulation of germtube formation in Candida albicans. Microbiology. 1987 Feb 1; 133(2): 41524.

Ruhnke M. Epidemiology of Candida albicans infections and role noncandida albicans yeasts. Current Drug Targets 2006; 7:495-504.

Sachin C Deorukhkar et al., Evaluation of Different media for germ tube production of Candida albicans and Candida dubliniensis. IJBAR (2012) 03 (09).

Sachin C Deorukhkar et al., Evaluation of Different media for germ tube production of Cndida albicans and Candida dubliniensis. IJBAR (2012) 03 (09).
Sachin CD, Ruchi K, Santosh S. In vitro evaluation of proteinase, phospholipase and haemolysin activities of Candida species isolated from clinical specimens. Int J Med Biomed Res 2012; 1: 153-157.

Sheppard DC, Locas MC, Restieri C, Laverdiere. Utility of germ tube formation at $39^{\circ} \mathrm{C}$ Yeast 2002; 19: 957962.

Sullivan D, Haynes K, Bille J, Boerlin P, Rodero $\mathrm{L}$ et al., widespread geographical distribution of oral Candida dubliniensis strains in human immunodeficiency virus-infected individuals. J Clin Microbiol 1998; 36:3007-3012.

Taschdjian CL, Burchall JJ, Kozinn PJ. Rapid identification of Candida albicans by filamentation on serum and serum substitutes. Am J ClinPathol. 1960; 90: $212-215$

\section{How to cite this article:}

Aditi Mehta, Mritunjay Kumar, Upasana Bhumbla, Anamika Vyas and A.S. Dalal. 2018. Comparison of Different Media for Germ Tube Production by Candida albicans: A Retrospective Study. Int.J.Curr.Microbiol.App.Sci. 7(06): 819-823. doi: https://doi.org/10.20546/ijcmas.2018.706.096 